\title{
Orientalismus und Ossianismus. Zu den Verschränkungen der Nord- und Orient- Diskurse in der polnischen Frühromantik
}

\author{
HEINRICH KIRSCHBAUM
}

\section{DOPPELGÄNGERTUM DER DISKURSE UND PARTIALITÄTSSCHREIBEN}

Eine der Besonderheiten der europäischen kulturellen und politischen Diskurse Ende des 18. bzw. Anfang des 19. Jahrhunderts bestand unter anderem in der beschleunigten Konzeptualisierung des geographischen Raumes und des Klimas des Landes; ${ }^{1}$ Die zentrale Rolle bei solchen Geokulturosophemen, die man auch

1 Entscheidend hat zur Etablierung geopoetologischer Fragestellungen Susi Frank in ihren diversen Publikationen beigetragen (Grundlegendes bei FRANK, Susi: Überlegungen zum Ansatz einer historischen Geokulturologie. In: Zeit-Räume. Neue Tendenzen in der historischen Kulturwissenschaft aus der Perspektive der Slavistik. Hg. v. DERS. Wien 2002 (= Wiener Slawistischer Almanach 49, 2002), 55-75 und DIES.: Geokulturologie und Geopoetik: Definitions- und Abgrenzungsvorschläge. In: Geopoetiken: Geographische Entwürfe in den mittel- und osteuropäischen Literaturen. Hg. v. Magdalena MARSZAŁEK und Sylvia SASSE. Berlin 2010 (Literaturforschung 10; TopographieForschung 1), 19-43). Von dem wachsenden Interesse für die ostslawischen Geound Klima-Diskurse zeugen neben dem oben erwähnten Sammelband von MARSZALEK und SASSE eine Reihe von Artikeln in der Zeitschrift „Novoe literaturnoe obozrenie“ (Nr. 99, 2009): ANISIMOV, Kirill: Klimat kak „Zakosnelyj separatist“. Simvoličeskie i političeskie metamorfozy sibirskogo moroza [Das Klima als „steifer Separatist". Symbolische und politische Metamorphosen des sibirischen Frosts]. In: Novoe literaturnoe obozrenie 99 (2009), 98-114. - BogDANOV, Konstantin: Klimatologija russkoj kul'tury. Prolegomen [Klimatologie der russischen Kultur. Prolegome- 
als Nationalisierungen des Geographischen im Allgemeinen und der Himmelsrichtungen im Besonderen bezeichnen könnte, kommt dem Norden und dem Osten bzw. dem Orient zu. Den wichtigsten rhetorisch-poetischen Raum für die Konstruktionen der nationalen Geoidentität bildete dabei die Dichtung. In den Literaturen der jeweiligen Länder, die sich nun zunehmend als Nationalliteraturen selbst definieren, erhält der Diskurs des Nationalen mitsamt seiner geohistorischen und geopolitischen Komponente seine Prägung. Dabei kommt es zu paradox anmutenden Erscheinungen: Zum einen wandern romantische Topoi und Motive, welche zu Mythologemen und Ideologemen werden, von Land zu Land, von einer (poetischen) Kultur in die andere. Zum anderen aber wird dieses interkulturelle und interliterarische Gemeingut zur Selbstspezifizierung eingesetzt, was eine Abgrenzung von den Literaturen und Kulturen der Nachbarländer impliziert.

Einen der relevantesten Diskurse, in denen sich die europäischen (Literatur-) Kulturen definieren, bildet zu Beginn des 19. Jahrhunderts der poetische Orientalismus, hier auch im postkolonialistischen, Saidschen Sinne interpretiert. Im Falle Mickiewicz' wird das poetische und zugleich politische Konstrukt Litauen zu einer solchen Orientalisierungs- und Selbstorientalisierungsfläche. Dirk Uffelmann, der in einem Artikel die Beschreibungen der (anti-)kolonialen Doppelstimmigkeitsstrategien in Mickiewicz' „Konrad Wallenrod“ (1828) untersucht, vertritt die Auffassung, dass der Befund, Litauen bleibe für den sich gewaltsam relithuanisierenden Verräter [Konrad - H.K.] ein unerreichbares, hybrides Gebilde, das über den Helden hinausreiche. Mit Adam Mickiewicz (1798-1855) haben wir es, so Uffelmann, mit einem in Litauen geborenen, in der polnischen Kultur sozialisierten, nach Russland verbannten Schriftsteller zu tun, der später, im Pariser Exil, das Werk („Pan Tadeusz“) schreiben würde, das Litauen zum hybriden Ort der polnischen Seele erhebt. Das Litauische habe - als eigenes Fremdes - einen festen Platz auf der mental map der polnischen Kultur. Die Überlegung, warum Mickiewicz Konrad von Wallenrode nicht auch eine polnische Herkunft andichten konnte und warum das eigene Fremde der polnischen Kultur das Litauische sein musste, beantwortete Dirk Uffelmann damit, dass Litauen die Funktion eines eigenen ,Orients' der polnischen Kultur übernehmen konnte. Fern und doch vertraut, mit dem Eigenen (Katholischen) verbunden und doch - im 14. Jahrhundert - noch heidnisch und fremd. Die dosierte Fremdheit prädestiniere Litauen als polnisches eigenes Fremdes für das doppelstimmige

non“]. In: Ebd., 60-97. - KeLLI, Katriona: Sorok sorokov doždej: kak delali „peterburgskuju pogodu“ [Vierzig mal vierzig Regen: Wie wurde das „Petersburger Wetter“ konstruiert]. In: Ebd, 115-138. 
Sprachrohr des Verräters, der innen sei und zugleich außen, in zivilisierte Gewänder gekleidet und doch gleichzeitig ein wild kämpfendes Tier. ${ }^{2}$ Wie uns scheint, bedürfen die zutreffenden Analysen und Schlussfolgerungen Uffelmanns, der Mickiewicz' Litauen die Funktion des Orients attestiert, einiger kleiner Nuancierungen bzw. Ergänzungen.

Die Besonderheit der Situation Polens bestand darin, dass es nach 1795 keine Eigenstaatlichkeit mehr besaß und zwischen Preußen, Österreich und Russland aufgeteilt war. In der Literatur des geteilten und somit auch in gewissem Sinne entgeographisierten Polen entsteht ein romantisch geprägtes, poetisch-politisches Konstrukt ,Polen“, das in das geokulturosophische und geopolitische Koordinatensystem Europas neu eingeschrieben wird. Dabei verschränken sich die diese Konstruktionen tragenden Diktionen mit den aktuellen europäischen und somit auch von der polnischen (literarischen) Kultur zu rezipierenden poetischen und kulturellen Diskursen.

Das Bewusstsein dieser geteilten (Spalt-)Lage Polens bestimmt maßgeblich die Modi der Fremd- und Selbstdefinitionen Polens. Sowohl innere polnische Diskurse der polskość als auch fremde (bzw. feindliche) Polen-Konstrukte imaginieren (und/oder diskreditieren) die vergangene (,historische') und potenziellprovidenzielle, künftige Integrität Polens über seine Partialität. Die entscheidende Rolle kommt bei diesen Pars-pro-toto-Narrativen, die man auch ,äsopisches Partialitätsschreiben' nennen könnte, diversen Metonymien bzw. Antimetonymien Polens (z.B. in den Kreuzritternarrativen) $\mathrm{zu}^{3}$ Die damit verbundenen komplexen und oft widersprüchlichen Orientalismen und Selbstorientalisierun-

2 Uffelmann, Dirk: „Litwo! Wschodzie mój!“ [Litauen! Mein Orient!] In: Słupskie Prace Filologiczne. Seria Filologia Polska 8 (2010), 165-188.

3 Zum negativen Deutschenbild, entstanden durch die romantische Thematisierung der Kreuzritterthematik, vgl. ZYBURA, Marek: Krzyżak. Zur Entstehung des negativen Deutschenbildes in der polnischen Romantik. In: Narrative des Nationalen. Deutsche und polnische Nationsdiskurse im 19. und 20. Jahrhundert. Hg. v. Izabela SURYNT und Marek ZYBURA. Osnabrück 2010 (Studia Brandtiana 2), 147-158. Zu den Kreuzritternarrativen in Preußen vgl. SURYNT, Izabela: Sendungsbewusstsein und Kolonialräume. Die Kreuzritter im preußisch-deutschen Diskurs der zweiten Hälfte des 19. Jahrhunderts. In: Ebd. 181-206. Zum polnischen und deutschen Erinnerungsort Tannenberg vgl. PRZYBUŁA, Piotr: 1410, „gedächtnisfrisch“. Deutsche und polnische Tannenberg-/Grunwald-Imaginationen zwischen Geschichte und Gedächtnis. In: Ebd., 159-180. Zu Tannenberg als Erinnerungsort vgl. auch: SCHENK, Benjamin Frithjof: Tannenberg/Grunwald. In: Deutsche Erinnerungsorte. Hg. v. Etienne FrANÇOIS und Hagen SCHULZE. Bd. 1, Berlin 2005, 438-454. 
gen (seien es Selbstukrainisierungen oder Selbstlithuanisierungen wie in „Konrad Wallenrod") geraten ihrerseits in den Kontext der aktuellen europäischen und amerikanischen literarischen Mode- und Modelldiskurse (wie der Indianer-, der Janitscharendiskurs u.a.), in die nun die polnischen Konstellationen projiziert werden.

Mickiewicz' Litauen wird demnach nicht nur zu dem poetisch und politisch (antiimperial, antikolonial) ersuchten Geokulturosophem, in dem Selbstorientalisierungsstrategien Polens verhandelt werden. Bei der Konstruktion Litauens als der besseren Metonymie Polens (sowohl historisch-revisionistisch als Teil der Rzecz Pospolita als auch ahistorisch-nostalgisch als verlorenes, heidnisches und somit natürlicheres Eigenes) haben wir es auch mit den Konstruktionen zu tun, die man hier provisorisch ,Nordismen' nennen könnte. Der Begriff ,Nordismus' ist zwar belegt und ist etwa gleichbedeutend mit dem Panskandinawismus, dieser selbst scheint aber ein späteres Nebenprodukt der im 19. Jahrhundert stattfindenden Politisierung früherer Nord-Diskurse zu sein, die in der Literatur des 18. Jahrhunderts ausgetragen wurden.

Für die (prä-)romantische Literatur scheint dabei vor allem James MacPhersons (1736-1796) Ossian-Dichtung Muster und Modelle entwickelt zu haben. Die englische Dichtung konstruiert mit der Feder des Schotten MacPherson das verfremdende und verfremdete Eigene in der erdachten gälischen bzw. keltischen Kultur. Man kann den Einfluss der Dichtung des „Homer des Nordens“ sowohl auf die für die slawische Selbst(er-)findung paradigmatischen Volkstümlichkeits- und Slawenkonzepte von Herder als auch auf die für die slawischen poetischen Kulturen ebenfalls einflussreichen Nord-Konstrukte von Madame de Staël kaum überschätzen. ${ }^{4}$ Bei den hier interessierenden Mechanismen handelt es sich nicht nur um die Motive der ossianischen Poetik ${ }^{5}$ - auch wenn diese für

4 Vgl. zur Ossian-Rezeption in Europa: The Reception of Ossian in Europe. Hg. v. Howard GASKILL. London 2004 (The Athlone critical traditions series 5); speziell zur französischen Rezeption VAN TIEGHEM, Paul: Ossian en France. 2 Bde, Genève 1967 (Nachdruck der Ausgabe Paris 1917), zum deutschen Ossianismus vgl. TOMBO, Rudolf: Ossian in Germany: bibliography, general survey, Ossian's influence upon Klopstock and the bards. New York 1966 (Columbia University germanic studies 2) [Nachdruck der Ausgabe New York 1901]; zum russischen Fall vgl. LeVIN, Jurij: Ossian v russkoj literature [Ossian in der russischen Literatur]. Leningrad 1980. Zur Rezeption des Ossian-Stoffes in der Kunst vgl.: Ossian und die Kunst um 1800. Ausstellungs-Katalog Kunsthalle Hamburg 1974. München 1974.

5 Vgl. hier aber auch die Konstrukte des Wilden bzw. der Wildheit. Zur Topik des erhabenen Wilden in den Ossian-Liedern MacPhersons vgl. STAFFORD, Fiona J.: The Sub- 
Jahrzehnte zu den Topoi der gesamteuropäischen Literatur werden -, sondern auch um das Prinzip der Erfindung der gesuchten ,authentischeren“ poetischen und kulturellen (Ur-)Heimat in der nahen, ja nächsten Nachbarkultur selbst. Dieses Prinzip könnte man in Analogie zum Orientalismus ,Ossianismus‘ nennen.

Nach dem ,ossianischen', englisch-schottischen Muster suchen die anderen europäischen Literaturen nach ihrem wieder zu entdeckenden Eigenen im Allgemeinen und nach ihrem eigenen ossianischen Norden im Besonderen. Bei der Sehnsucht nach dem Norden handelt es sich zudem um eine Art geopoetisch umgedrehte neoklassizistische Sehnsucht nach dem seligen Süden, den man, der terminologischen Poetik der Ismen folgend, auch ,Mediterranismus ' bezeichnen könnte. Mediterranismus und Ossianismus existieren und behaupten sich in Interaktion und in reziproker diskursiver Dependenz voneinander; dabei überlappen sie sich auch mit denjenigen des Orientalismus. Viele Topoi und Heterostereotype des Mediterranismus werden zum einen auf den Orient übertragen, zum anderen beinhaltet der Orientalismus historisch und aktuell-politisch die griechische und somit auch eine (anti-)osmanische, d.h. (anti-)orientale Komponente (man denke dabei an die Blütezeit des Philhellenismus sowie auch an die hier zur Debatte stehenden 1810er und 1820er Jahre). Mediterranismus, Ossianismus und Orientalismus interferieren und konkurrieren, sie ahmen gegenseitig ihre rhetorisch-diskursiven Schemata nach und sie trans- und deformieren sie zugleich auch. Zusammen bilden sie ein komplexes interdiskursives Feld, dessen Diktionen, Argumentationsfiguren und motivisch-metaphorische Strukturen einander sowohl komplimentieren als auch herausfordern. Dabei kommt es zu entsprechenden Verschränkungen, Verschiebungen und Umkodierungen, welche von den anderen Diskursen rezipiert und in diversen Revisionsverfahren aufs Neue adaptiert werden. Der Kampf bzw. Wettlauf um die eigene Autonomie und rhetorische Souveränität der einzelnen Diskurse, sei es Orientalismus, Ossianismus oder Mediterranismus, setzt auch den Einsatz fremder diskursiver Mittel voraus.

Bei diesen reziproken Nachahmungen kommt es zu einem Phänomen, das man als Doppelgängertum der Diskurse bezeichnen würde und bei dem das Original sich schwer herausfinden bzw. unterscheiden lässt. ,Doppelgänger' ist hier natürlich eine Metapher; der Zugriff auf das metaphorische Feld des Doppelgängertums scheint allerdings legitim und zeitgerecht zu sein, nicht nur weil dieses Thema und seine Variationen zu den Topoi der romantischen Literatur im All-

lime Savage. A Study of James Macpherson and the Poems of Ossian. Edinburgh 1988. Diese Topik wird in der Romantik weitergeführt und an die ,eigenen', ,einheimischen' historischen und geokulturosophischen Mythen angepasst. 
gemeinen und der Texte Mickiewicz' im Einzelnen gehören, ${ }^{6}$ sondern auch weil es indirekt das hier interessierende Phänomen der Spaltung bzw. des Verlustes der romantischen Identität beinhaltet. Das Doppelgängertum von Diskursen und diskursiven Praktiken zeugt von demselben Problem auf der Ebene der nationalen Identitätsbildung dieser Zeit, im polnischen Fall korrespondiert es zudem mit dem oben angesprochenen Phänomen der nationalen (Persönlichkeits-)Spaltung (,drei Polen') und des Partialitätsschreibens.

In diesem Doppelgängerdialog der Diskurse gehört dem Ossianismus die erste Stimme. In den slawischen Literaturen werden ossianische Muster und ossianistische Verfahren unterschiedlich einverleibt. So wird in der russischen Literatur, etwa bei Konstantin Batjuškov (1787-1855), zunächst die Handlung der neo-ossianischen Elegie noch im skandinavischen Norden bzw. in Deutschland verortet, ${ }^{7}$ während die nachfolgende Generation bereits, etwa in Evgenij Baratynskijs (1800-1844) „Finlandija“ [Finnland], ihren eigenen Norden entwirft. ${ }^{8} \mathrm{Im}$ nächsten Schritt erfolgt die Nordisierung des Eigenen. Hierbei konkurrierten germanische und slawische (russische und polnische) Literaturen darum, der

6 Vgl. in dieser Hinsicht nicht nur die Variationen des Doppelgängermotivs in der Figur bzw. in den Figuren des Hauptprotagonisten von „Konrad Wallenrod“, sondern auch das ihm semantisch angrenzende balladeske Leitmotiv von Mickiewicz' Frühwerk: das des Vampirs/Gespenstes, das zwischen Lebenden und Toten wandert und wandelt und nicht zur Ruhe kommt.

7 Vgl. Batjuškovs „schwedische“ Elegie „Na razvalinach zamka v Švecii“ [Auf den Ruinen eines Schlosses in Schweden] und die „germanische“ „Perechod čerez Rejn“ [Die Überquerung des Rheins] (BATJUŠKOV, Konstantin N.: Opyty v stichach i proze [Versuche in Vers und Prosa]. Moskva 1978, 202-205, 320-324). Charakteristisch ist, dass der überzeugte Italophile Batjuškov auch stark mediterranistisch inspirierte Gedichte verfasste bzw. deren Topoi in seine nordistischen Meditationen einbaute. Die Sehnsucht nach dem ,seligen Süden' wird in seinem Werk (und später im 20. Jahrhundert bei Osip Mandel'štam) zur untrennbaren Komponente der Selbstnordisierung, und zwar sowohl als deren Ergänzung, als auch als deren Neutralisierung und Ausgleich.

8 Vgl. BaratynskiJ, Evgenij A.: Stichotvorenija. Poèmy [Gedichte. Poeme]. Moskva 1982, 7-10. Die russische Dichtung entdeckt das kurze Zeit davor in Folge des Russisch-Schwedischen Krieges von 1808-09 von Schweden übernommene Finnland als ein ossianisches bzw. ossianisierbares poetisches Nachbarland. Bei russischen Finnland-Poetisierungen wird dabei eine andere, für die Selbstossianisierungsstrategien relevante metonymische Verbindung aufgebaut: Finnland - Skandinavien - Schottland. 
einzig wahre Norden oder zumindest ein untrennbarer Teil dieses mit positiven poetischen Konnotationen aufgeladenen Nordens zu sein.

An dieser Auseinandersetzung war auch Adam Mickiewicz beteiligt, der sich sowohl der litauischen Geschichte (die Auseinandersetzung mit dem Deutschen Orden in „Konrad Wallenrod“) als auch der programmatisch ahistorischen, nicht zivilisierten, legendären litauischen Vergangenheit annimmt. Eine solche Erschaffung des fremden Eigenen, des eigenen Polnischen in der nächsten kulturellen Metonymie musste nicht unbedingt im direkten Norden sein. In der sogenannten Ukrainischen Schule der polnischen Poesie (Antoni Malczewski, Seweryn Goszczyński, Aleksander Groza, Józef Bohdan Zaleski, Michał Czajkowski, Tomasz Padurra, Wacław Seweryn Rzewuski) wird dieses ,wahre Polen“ nach dem gleichen Prinzip des Ossianismus, allerdings zusätzlich geprägt durch die präpanslawistische Rhetorik, in der imaginierten Ukraine verortet. ${ }^{9}$ In den 1820er Jahren beginnen die Diskursdoppelgänger des ersehnten Polentums zu interferieren und zu konkurrieren. Mickiewicz, der sich in seinem ,nordistischen“, ,litauischen' Schreiben u.a. auch indirekt gegen die in den 1820er Jahren aufsteigende Ukrainische Schule wendet, versucht, sich selbst als MacPherson bzw. Ossian Litauens zu etablieren und somit für sich den Lorbeerkranz des polnischen Nationaldichters zu beanspruchen. Das zu orientalisierende und zugleich $\mathrm{zu}$ ossianisierende (nordisierende) Litauen wird dabei zum multifunktionalen diskursiven Feld, auf dem neben der Selbstbehauptung der eigenen, polnischen Romantik im gesamteuropäischen Kontext auch die Schlacht um den Titel eines polnischen Homers bzw. Ossians ausgetragen und - wenn man sich den Stellen-

9 Vgl. KIRČIV, Roman F.: Ukraïns'kyj fol'klor u pol's'kij literaturi [Die ukrainische Folklore in der polnischen Literatur]. Kiiv 1971. Vgl. in diesem Kontext auch Michał Kuziaks Überlegungen zum Alteritätsdiskurs in der polnischen Literaturkritik am Beispiel Brodzińskis, Mickiewicz' und Mochnackis. In seinem Beitrag geht Kuziak der Kategorie der Differenz in der polnischen Romantik nach. Alterität werde im Paradigma der historischen Nationalcharaktere entwickelt. Dabei komme es zu oxymoral anmutenden Versuchen, Pluralisierung und Differenzierung mit innerer Einheit zu verbinden. So wird die Konzeption des Idyllischen bei Brodziński mit slawischen Mythen verknüpft, während Mochnacki mit der nordischen Mythologie argumentiert und Mickiewicz nach einer Ausbalancierung zwischen den romantischen Süden- und Osten-Selbsteinschreibungen sucht (KUZIAK, Michał: Brodziński, Mickiewicz, Mochnacki: Der Alteritätsdiskurs der polnischen romantischen Kritik. In: Romantik und Geschichte. Polnisches Paradigma, europäischer Kontext, deutsch-polnische Perspektive. Hg. v. Alfred GALl u.a. Wiesbaden 2007 (Veröffentlichungen des NordostInstituts 8), 173-183, hier 176-179). 
wert Mickiewicz' in der Rezeption der polnischen Literatur vergegenwärtigt schließlich für letzteren entschieden wird.

Freilich bestimmten die ossianistischen Verfahren bereits das poetische Verhalten vor Mickiewicz. Ein Beispiel dafür stellt die Literaturgeschichte des sogenannten duma-Genres in den ersten Jahrzehnten des 19. Jahrhunderts dar. Die Tradition der polnischen duma entsteht ebenfalls maßgeblich durch Übernahmen und Übertragungen ossianistischer Muster. Direkte Assoziationen mit den ähnlichen Gattungen aus dem 17. Jahrhundert führen eher in die Irre. Laut Zgorzelski wurde die Gesangstradition von Kochanowski, Szarzyński und anderen Dichtern des 16. und 17. Jahrhundert in dieser Zeit offensichtlich als zu schwach empfunden, so dass weder Niemcewicz noch ein anderer zeitgenössischer Dichter an sie anknüpft. Es ginge um etwas Anderes, um das Lied, das die heroischen Motive mit denjenigen der Liebe vereinen würde: um das populäre Volkslied nach dem Ossian-Muster. Auf dieser Plattform berührten die ersten dumy Niemcewicz' die Tradition der ukrainischen dumy, die im 17. Jahrhundert so populär an den Höfen des (Klein-)Adels waren. ${ }^{10}$

Die Erschaffung der eigenen, einheimischen, nostalgisch kolonialen dumaSujets geht in der polnischen Literatur am Anfang des 19. Jahrhunderts Hand in Hand mit der Übernahme bzw. Nachahmung europäischer (poetischer) Muster und Motive. Die Gattung der duma bietet im ersten Jahrzehnt des 19. Jahrhunderts Raum für direkte bzw. offene Übertragungen ossianistischer Balladen. So nennt Julian Ursyn Niemcewicz (1757-1841) seine Nachdichtung der Ballade „Alonzo the Brave and Fair Imogine“ von Matthew Gregory Lewis (1775-1818) noch „Alondzo i Helena. Duma naśladowana z angielskiego“ [Alonzo und Helene. Duma, nachgedichtet (nachgeahmt) aus dem Englischen]. ${ }^{11}$ Die Tatsache, dass Niemcewicz des Englischen mächtig war, begünstigte den direkten Kontakt mit dem englischen Post-Ossianismus unter Auslassung der deutschen Zwischenstation (obwohl Lewis' "Alonzo“ in Wirklichkeit eine Variation bzw. Nachdichtung von Gottfried August Bürgers „Lenore“ darstellt), zumindest in der Wahrnehmung Niemcewicz'. ${ }^{12}$ Ossianistische motivische Spuren (Abschied

10 ZGORZELSKI, Czesław: Duma popredniczka ballady [Duma als Vorläuferin der Ballade]. Toruń 1949, 32.

11 NiemCEWICZ, Julian Ursyn: Pisma różne wierszem i prozą [Diverse Schriften in Versen und Prosa]. Tom I. Warszawa 1803, 491. Vgl. auch Niemcewicz’ duma „Sen Marysy“ (DERS.: Bajki i powieści. [Fabeln und Erzählungen] Bd. 1. Warszawa 1820, 173f.).

12 Zum Einfluss der deutschen Literatur auf Lewis vgl. CONGER, Syndy M.: Matthew G. Lewis, Charles Robert Maturin and the Germans. An interpretative study of the influ- 
der Verliebten in Folge eines Krieges, Klage über den Tod des/der Geliebten am Grabe etc.) lassen sich auch in Bruno Kicińskis (1794-1844) duma „Ludmila w Ojcowje ${ }^{\text {"13 }}$ nachweisen, hier allerdings lokalisiert bereits im slawischen Kontext. $^{14}$

Rudimente dieser Topik sind auch im orientalistischen Schreiben Mickiewicz' zu finden, wie z.B. im Krimsonett „Grób Potockiej“ [Potockas Grab] (1826), in dem der verbannte polnische Dichter sich mit der im Harem gefangengehaltenen Maria Potocka identifiziert und in einer neo-ossianistischen Pose sein und pars pro toto auch das gesamtpolnische Schicksal des/der Gefangenen beweint. Dabei vermischen sich post-ossianische Selbstnordisierungen mit Orientalismen und Selbstorientalisierungsstrategien. Mickiewicz' romantische Erfindung Litauens, sei es in „Ballady i romanse“ [,Balladen und Romanzen“] (1822-23) oder in den „Dziady“ II und IV, vollzieht sich über die ossianistische Selbst-Metonymisierungsroute. Dabei mag Mickiewicz auf die Bezeichnung duma nicht nur verzichtet haben, weil er die für die polnische Literatur neuere,

ence of German literature on two gothic novels. Salzburg 1977 (Salzburg studies in English literature. Romantic reassessment 67). Zum Verhältnis zwischen Lewis' Original und Niemcewicz' Nachdichtung s. KLEINER, Juliusz: Sentymentalizm i preromantyzm. [Sentimentalismus und Präromantik]. Kraków 1975, 37-46.

13 KICIŃSKI, Bruno: Ludmila w Ojcowje. Duma [Ludmila in Ojców. Duma]. In: Tygodnik Polski i zagraniczny 35 (1818), 193-196.

14 Vgl. auch WęŻYK, Franciszek: Duma o Wandzie [Duma über Wanda]. In: Pszczółka Krakowska 48/13 (1820), 201-205. Wężyk hat auch 1810, inspiriert nicht zuletzt durch Niemcewicz’ Gliński-duma, das Drama „Gliński“ geschrieben (DERS.: 1821. Gliński. Kraków 1821). Eine slawische Kontextualisierung konnte auch russische Konnotationen beinhalten. Vgl. die Übertragung der ossianischen Topik in den russischen Kontext in Niemcewicz' „Okropna Puszcza“ [Schrecklicher Urwald] (NIEMCEWICZ, Bajki, 1820 (wie Anm. 11), 199f.). Vgl. die Klassifikation der Multifunktionalität der Bezeichnung duma und deren poetischer Inhalte in der polnischen Literatur der 1800-1820er Jahre im Allgemeinen und bei Niemcewicz im Besonderen bei ZGORZELSKI (wie Anm. 10), 35-38. Dass Niemcewicz die Bezeichnung der , alten neuen' Gattung und deren Topoi noch inkonsequent gebraucht, bezeugt die Tatsache, dass er in seine „Pisma rożne wierszem i prozą“ auch „Romans o miłościach Medzuna Leili“ [Romanze über Meğnūn-Lailās Liebschaften] aufnimmt, die Romanze, die er duma perska nennt (vgl. NIEMCEWICZ, Julian Ursyn: Pisma różne wierszem i prozą [Diverse Schriften in Vers und Prosa]. Bd. 2. Warszawa 1805, 336-348). Dabei dichtete Niemcewicz die persische duma nach einer französischen Vorlage nach. Vgl. ZGORZELSKI (wie Anm. 10), 37. 
aktuellere und europäischere Bezeichnung Ballade wählte (und sich somit als europäischer Dichter postulieren konnte), ${ }^{15}$ sondern auch, weil die Bezeichnung duma zu sehr polnisch-ukrainische Assoziationen hervorgerufen hätte, während er vor der Aufgabe stand, eine polnisch-litauische poetische Identität zu kreieren. Die direkt aus dem Ossianismus stammende, „nordische“, d.h. auch selbstnordisierende Ballade passte dazu viel besser. ${ }^{16}$ Außerdem war die Bezeichnung duma zu sehr mit Niemcewicz konnotiert, aus dessen Schatten Mickiewicz heraustreten wollte. Den polonozentrischen identitätsstiftenden Schreibstrategien Niemcewicz' (z.B. bei der Wahl der slawischen Bezeichnung duma anstelle der Ballade $)^{17}$ setzte Mickiewicz eine selbst-europäisierende und zugleich selbstnordisierende entgegen. Die Selbst-Nordisierung bzw. Selbst-Ossianisierung, die auch Selbst-Orientalisierung bedeutet, implizierte dabei auch eine Selbsteuropäisierung. Der anscheinende Umweg über die litauische Metonymie erwies sich schließlich als effektiverer Zugang zum Polentum als der in der poetischen Generation vor Mickiewicz propagierte direkte Weg.

Eine ganz andere Revision des Einflusses Niemcewicz' gab es bei den Vertretern der sogenannten Ukrainischen Schule. Auf die Bezeichnung duma und die ukrainische Verortung literarischer Konstrukte wird nicht verzichtet, aber es kommt zu einer zunehmenden Eliminierung der ossianistisch-sentimentalistischen und somit fremden, europäischen Note der dumy. An ihre Stelle tritt die ,orientalische“ ,Janitscharenbrutalität“ der Kosakenbilder, angefangen mit Malczewskis „Maria“ und fortgesetzt bei Zaleski, Goszczyński u.a. Die SelbstUkrainisierung bzw. Selbstkosakisierung nimmt dabei viel explizitere selbsto-

15 Zur Krise der Bezeichnung duma wegen des Aufkommens der Bezeichnung ballada vgl. ZGORZELSKI (wie Anm. 10), 63f.

16 Dumy über Litauen sind rar. Eine Ausnahme bildet Dumanie u rozwalin zamku Giedyminu von Mickiewicz' Wilnaer Freund und Philomaten Onufry Pietraszkiewicz (1793-1863), der zusammen mit Mickiewicz nach Russland verbannt wurde (vgl. den Text des Dumanie in: CzuBeK, Jan: Poezja filomatów. Bd. 1. Kraków 1922, 156160). Hier tritt aber dumanie in der Bedeutung ossianistischer Meditation bei der Betrachtung der Ruinen alter Schlösser und Burgen auf. Diese ossianistische Topik durchdringt nicht nur die dumy mit der Krakauer Kulisse, sondern auch einige ,ukrainische“ Texte der Epoche. Vgl. das Gedicht Duma na gruzach Ojczyzny [Duma auf den Trümmern des Vaterlandes] von Seweryn Goszczyński (vgl. ZGORZELSKI (wie Anm. 10), 57f.).

17 Zur Wahl der Bezeichnung duma anstelle der Ballade bei Niemcewicz vgl. auch ZGORZELSKI (wie Anm. 10), 35f. 
rientalisierende Züge an als es in den Poetisierungen der Nord-Metonymien Polens der Fall ist.

Ein Paradebeispiel für ossianistisch-orientalistische (anti-)koloniale Umkodierungen stellen in diesem Kontext polnische und russische Nachdichtungen der postossianistischen deutschen Ballade dar. Die neue (Wilnaer) Dichtergeneration, der auch Mickiewicz angehört, eignet sich die postossianistischen Verfahren über die deutschen Kanäle an. Zum Schlüsseltext dieser Aneignungen wird Bürgers „Lenore“. Während Niemcewicz in „Malwina“ „Lenore“ über ihre englische Variante (Lewis) adoptiert ${ }^{18}$, rezipieren die jungen Wilnaer Literaten den Kulttext Bürgers zunächst über eine russische Zwischenstation - über Vasilij Žukovskijs Nachdichtung -, was entsprechende essentielle Revisions- und Rivalitätseffekte in der Rezeption nach sich zieht. Es gehört zu den brisanten, wenn auch der Logik des Streites um neue und alte poetische und kulturelle Identitäten nicht widersprechenden Beispielen der reziproken Etablierung der russischpolnischen Romantik, dass die polnische Begeisterung für die Ballade durch die Lektüre eines russischen Textes ausgelöst wurde. ${ }^{19}$ Edward Odyniec, der zwei-

18 Vgl. auch die sich bereits auf die deutsche Vorlage stützenden Nachdichtungen von Krystyn Lach Szyrma (SzYRMA, Krystyn Lach: Kamilla i Leon. Naśladowanie Leonory Bürgera [Kamilla und Leon. Nachahmung (Nachdichtung) von Bürgers Lenore]. In: Pamiętnik Naukowy służący za ciąg dalszy ćwieczeń naukowych. Oddział literatury. Bd. 1. Warszawa 1819, 358-366) und Julian Bogucki (BoGUCKI, Julian: Halina (Ballada z Bürgera) [Halina. Ballade nach Bürger]. In: Rozmaitości, Nr. 40, 06.10.1824, 315-317).

19 Vgl. DernaŁowicz, Maria/Kostenicz, Ksenia/MaKowiecKa, Zofia: Kronika życia i twórczości Mickiewicza: Lata 1898-1824. Warszawa 1957, 120; oder KoŚNY, Witold: Adam Mickiewicz und Gottfried August Bürger. In: Mickiewicz und die Deutschen. Hg. v. Eva MAZUR-KeBLOWSKI. Wiesbaden 2000 (Veröffentlichungen des Deutschen Polen-Instituts Darmstadt 13), 67-87, hier 69f. Zu polnischen und russischen Bürgerübersetzungen vgl. ZGORZELSKI (wie Anm. 10), 78-79. - KOŚNY, Witold: Die polnischen Übersetzungen von G. A. Bürgers „Lenore“ vor dem Erscheinen der Ballade „Ucieczka“ von Adam Mickiewicz. In: Między oświeceniem i romantyzmem. Kultura polska około 1800 roku. Hg. v. Zdzisław LICHAŃSKI. Warszawa 1997, 255-272; und SUCHANEK, Lucjan: Wątek Lenory w balladzie polskiej i rosyjskiej (Mickiewicz, Żukowski, Katienin) [Das Lenore-Motiv in der polnischen und russischen Ballade (Mickiewicz, Žukovskij, Katenin]. In: Spotkania literackie: z dziejów powiązań polsko-rosyjskich w dobie romantyzmu i neoromantyzmu. Hg. v. Bohdan GALSTER. Wrocław 1973, 45-59. 
malige Übersetzer von Bürgers „Lenore“20 ${ }^{\text {20 }}$ und zugleich Übersetzer von Žukovskijs Bürger-Variationen, erinnerte sich gerne an den Abend, an dem Žukovskijs Ballade im Wilnaer Literatenkreis vorgelesen und kurz darauf zum Objekt zahlreicher Nachahmungen wurde. ${ }^{21}$

Žukovskij selbst übersetzte „Lenore“ dreimal: 1808 verfasste er seine Lenore-Nachdichtung unter dem Titel „Ljudmila“, 1808-12 unter dem Titel „Svetlana“, und schließlich 1831 als „Lenora“. In Bürgers „Lenore“ spielt die Handlung in „Deutschland“ bzw. in der deutsch-böhmischen Geschichte:

Er war mit König Friedrich's Macht

Gezogen in die Prager Schlacht. ${ }^{22}$

In seiner Nachdichtung russifiziert und nordisiert bzw. ossianisiert Žukovskij nicht nur den Namen der Hauptprotagonistin (Lenore wird zu Ljudmila), sondern auch den Handlungsort. Das Grab des Helden befindet sich im polnischen Litauen, am Narew:

„Близ Наревы дом мой тесный [...]“
„Где ж, скажи, твой тесный дом?““-
„Там, в Литве, краю чужом...“.”.

[,Nicht weit vom Narew ist mein enges Haus“ [...] // „Wo, sag mir, ist dein enges Haus?“ „Dort, in Litauen, in der Fremde..."]

Es ist dabei anzunehmen, dass Odyniec, Mickiewicz und deren Freunde nicht nur von der brandaktuellen und neuen romantischen Poetik von Žukovskijs Nachdichtung und von der Möglichkeit eines solchen romantischen Schreibens in einer slawischen Sprache fasziniert waren, sondern nicht zuletzt auch von der „litauischen“ Verortung der Erzählung. Das Bürger'sche Sujet wurde von Žu-

20 Vgl. Odyniec' Bürger-Übertragungen (ODYNIEC, Edward: Adela. Ballada z Bürgera [Adela. Ballade nach Bürger]. In: Dziennik Wileński 19.06.1822, II, Nr. 6, 213-223 und DERS: Poezje. Bd. 1. Wilno 1825, 3-16).

21 DERS.: Listy z podróży. Bd. 1. Warszawa 1961, 265 f.

22 BÜRGER, Gottfried August: Lenore. London 1796, 1.

23 ŽUKOVSKIJ, Vasilij: Sočinenija v trech tomach [Werke in drei Bänden] Bd. 2. Moskva 1980, 10f. Die Übersetzungen ins Deutsche sind, wenn nicht anders angegeben, von Heinrich Kirschbaum. 
kovskij im Kontext der polnisch(-litauisch)-russischen Kriege lokalisiert. ${ }^{24}$ Das Paradigma wurde vorgegeben, außerdem klang die litauische Färbung der Ballade wie eine indirekte Herausforderung an die polnische Dichtung, ihre LitauenPoetisierungen zu intensivieren, wenn sie will, dass Litauen, das bereits politisch zu Russland gehört, nun auch als poetisches Thema nicht zur Domäne der russischen Literatur wird.

In seinem Gedicht „Lilie“, das eine freie Variation der „Lenore“ darstellt, übernimmt Mickiewicz Žukovskijs Verfahren, verschiebt jedoch die geohistorische Achse. Der Held der Geschichte zieht mit Bolesław gegen die Kiewer (Metonymie für Russland) in den Krieg:

Mąż z królem Bolesławem

Poszedł na Kijowiany. ${ }^{25}$

[Mein Mann ist mit dem König Bolesław / Gegen die Kiewer gezogen.]

In Mickiewicz’ Nachdichtung „Ucieczka“ wird das Bürgersche Sujet, ähnlich zu Tomasz Zans Neryna, ebenfalls lithuanisiert. Inspiriert von der deutschen, Bürgerschen Ballade und deren russischen Adaptation legen die Wilnaer Literaten das Fundament, worauf in den nächsten Jahren der polnische poetische Lithuano-Ossianismus aufbauen wird.

Währenddessen arbeitet die russische Dichtung weiter an den litauischen Lokalisierungen der balladesken Sujets. Eine Variation der Bürger-Žukovskijschen Ballade und eine Art Nachdichtung aus dem ,Russischen ins Russische“ bildet das Fragment gebliebene Gedicht Kondratij Ryleevs „Ljudmila“, in dem der Hauptheld Milovid seinen Tod im fernen Litauen voraussagt:

Там далёко, за Днепром,

В Литве, на чужбине,

Кончу в бое я с врагом

Дни свои в кручине. ${ }^{26}$

24 Žukovskijs Opponent in der Diskussion um die Ballade, Pavel Katenin, lokalisiert die Bürgersche Geschichte im Nordischen Krieg: KATENIN, Pavel A.: Izbrannye proizvedenija [Ausgewählte Werke]. Moskva-Leningrad 1965, 91.

25 Mickiewicz, Adam: Dzieła [Werke]. Bd. 1. Warszawa 1955, 157.

26 RYLEeV, Kondratij F.: Polnoe sobranie stichotvorenij [Vollständige Gedichtsammlung]. Leningrad 1971, 300. 
[Dort, weit weg, hinter dem Dniepr, / In Litauen, in der Fremde, beende ich im Kampf gegen den Feind / meine Tage in Trauer.]

Ryleev schreibt seinen Text nach dem Paradigma von Žukovskij. Bezeichnend ist die Polonisierung der Betonung: в Л $и$ тве [in Litauen]. ${ }^{27}$ Charakteristisch ist auch die hier wie auch im polnischen (u.a. Mickiewicz'schen) Kontext vorhandene (ukrainische) Achse Dniepr - Litauen. Ryleev, der künftige Freund Mickiewicz' in der Zeit der Verbannung des letzteren in Russland, begeisterte sich für Mickiewicz' Poesie bereits Anfang der 1820er Jahre. Mickiewicz' BürgerVariation „Lilie“ kannte er sehr gut und versuchte sie sogar nachzudichten. ${ }^{28}$ In seinem ebenfalls nicht vollendeten Übersetzungsentwurf verzichtet Ryleev allerdings auf die Übersetzung der Mickiewicz'schen legendär-historischen Verortung der Erzählung in russisch-litauischen Kriegen. Auch der Hauptprotagonist heißt bei Ryleev, abweichend von Mickiewicz, russifiziert Dem'jan. ${ }^{29}$

Die russische Dichtung (genauso wie die polnische) kämpft im post- bzw. neoossianistischen Genre- und Themenkomplex nicht nur um Litauen, sondern auch direkt und indirekt um die Ukraine als politische und poetische Kolonie bzw. Metonymie Russlands (oder Polens). Schließlich wird „Litauen“ von der russischen Dichtung aufgegeben und dem poetischen „Feind“ überlassen. Die russische Literatur der 1820-30er Jahre (von Ryleevs „Dumy“ bis Gogol’s „Ta-

27 Hier mag Ryleevs Bekanntschaft mit der polnischen Sprache während seines Aufenthaltes im polnisch-litauischen Nieświż eine Rolle gespielt haben.

28 Vgl. RYLEEV (wie Anm. 26), 339-341.

29 War Mickiewicz' Verortung des Sujets im Kontext der russisch-polnischen Kriege ein Grund dafür, dass Ryleev die Übertragung des Textes unterbrochen hat? In jedem Fall steht Ryleevs Mickiewicz-Übertragung am Anfang seines widersprüchlichen Verhältnisses zu Polen und zur polnischen Literatur. Zur polnischen Komponente in Ryleevs Leben und Werk vgl. BERNDT, Margarethe: J. Niemcewicz und K. Ryleev. Berlin 1961. - DvojČEnKo-MARKova, E. M.: Nemcevič i Ryleev [Niemcewicz und Ryleev]. In: Pol'sko-russkie literaturnye svjazi [Polnisch-russische literarische Relationen]. Hg. v. Nikolaj BALAŠOv u.a. Moskva 1970, 129-155. - GALSTER, Bohdan: Twórczość Rylejewa na tle prądów epoki [Ryleevs Werk vor dem Hintergrund der Epoche]. Wrocław u.a. 1962, 54-59. - KIRSCHBAUM, Heinrich: Verrat, Revolte, Revision: Zu polnischen Komponenten in K. Ryleevs Werk. In: Die Welt der Slaven LVI/2 (2011), 335-359. - KotLJAREVSKIJ, Nestor: Ryleev. Sankt-Petersburg 1908, 80-82. MASLOV, Vasilij I.: Literaturnaja dejatel'nost' K. F. Ryleeva [K. F. Ryleevs literarische Tätigkeit]. Kiev 1912, 172-183; und SiROTININ, Andrej N: Ryleev i Nemcevič [Ryleev und Niemcewicz]. In: Russkij archiv 1 (1898), 67-82. 
ras Bul'ba") wird sich nun auf die literarische Einverleibung der Ukraine konzentrieren.

Für uns ist bei diesen Übertragungen und den sie begleitenden geopoetischen bzw. geohistorischen Umkodierungen nicht nur die Lokalisierung in der einheimischen und zugleich nordischen (wenn auch legendären) Geschichte von Relevanz, sondern auch die Wahl der Sujets aus den russisch-polnischen Kriegen, die den (para-)historischen Hintergrund der jeweiligen Erzählungen ausmachen und eine Art Umschreibung des russisch-polnischen literarischen Kampfes um neue poetische Themen und ihre geopoetischen Koordinaten bilden. Im Sujetrahmen der russisch-polnischen Kriege findet die Selbstreflexion der poetisch-thematischen und politischen Rivalität der beiden Literaturen und Kulturen statt. Die Selbstorientalisierungs- und Selbstnordisierungsdiskurse sowie Geokulturosopheme mitsamt ihrer Topoi und Sujetik werden nicht zuletzt zu den thematischen Flächen, in denen die jeweilige Literatur sich selbst diskursiviert. Die Metaebene der Literatur passt sich und knüpft an die jeweilige Dominante der Literatur an, in unserem Falle an die Themen und Topoi, die in den frühen 1820er Jahren, d.h. im Vorfeld des Dekabristen- bzw. Novemberaufstands - und diese gleichzeitig auch poetisch-ideologisch mit vorbereitend - eine zunehmende Politisierung erfahren. Für den hier interessierenden literaturhistorischen Abschnitt würde das Folgendes bedeuten: Einerseits vollzieht sich in der Literatur eine Ästhetisierung des Politischen, andererseits ist das Politische nur Material. Beim Eintreten in die Literatur gerät es in ein anderes Funktionalitätsnetz, es wird zu einem Metaverfahren der Literatur: Politisch-ideologisch motivierte und konnotierte poetische Geosopheme und Kulturosopheme werden von bzw. in der Literatur für eigene, auch metaliterarische Zwecke benutzt. ${ }^{30}$ In den genetisch internationalen und evolutionär nun nationale Identität stiftenden Narrativen beschreibt die (pol-

30 Vgl. KLINKERT, Thomas: Literarische Selbstreflexion im Medium der Liebe. Untersuchungen zur Liebessemantik bei Rousseau und in der europäischen Romantik (Hölderlin, Foscolo, Madame de Staël und Leopardi). Freiburg im Breisgau 2002 (Rombach-Wissenschaften/Reihe Litterae 92). Für die slawischen Kulturen, die mit Verspätung die Romantik rezipieren und domestizieren, findet diese Selbstreflexion bereits im Medium der politisierten bzw. zu politisierenden Diskurse statt. Zur Verstrickung des Poetischen und des Politischen in der Literatur vor den beiden Rebellionen (Dekabristen- und Novemberaufstand) vgl. auch GROB, Thomas: Literatur, Macht und politisches Ereignis. Der Dekabristenaufstand, der Polenaufstand und die ,Romantik‘. In: Imperium und Intelligencija. Fallstudien zur russischen Kultur im frühen 19. Jahrhundert. Hg. v. Jochen-Ulrich PETERS und Ulrich SCHMID. Zürich 2004 (Basler Studien zur Kulturgeschichte Osteuropas 9), 139-169. 
nische/russische romantische) Literatur sich selbst und ihren Kampf um den Platz unter der Sonne der europäischen Literaturen und, natürlich, den Kampf jedes einzelnen Schriftstellers um seine eigene souveräne Stellung in der jeweiligen Literatur.

\title{
DANDYS, PASChas, Renegaten. OSSIANISTISCH-ORIENTALISTISCHE DIKTIONEN IM FRÜHWERK ADAM MICKIEWICZ'
}

Der zweite Teil des vorliegenden Aufsatzes ist drei nicht ,klassischen“ Texten aus dem Frühwerk Adam Mickiewicz’ (vor den „Krimsonetten“) gewidmet, in denen die Verschränkung von Nord- und Orient-Diskursen erfolgte. Erste Ansätze der orientalistischen bzw. selbstorientalisierenden Diktion verbunden mit der Selbstverortung im Norden lassen sich noch vor der oben angesprochenen expliziten romantischen Phase, bereits im ersten publizierten Gedicht Mickiewicz', „Zima miejska“ [Der Stadtwinter] (1818) finden.

Mickiewicz' Wintergedicht beginnt mit einer Absage an die anderen Jahreszeiten, d.h. indirekt auch an die anderen poetisch und geokulturosophisch konnotierten Himmelsrichtungen. ${ }^{31}$ Nach dieser Abgrenzung beginnt die odische und zugleich elegisch meditative Kontemplation des Winters:

\author{
Witaj! narodom miejskim pora błoga, \\ Już i Niemeńców, i sąsiednich Lechów \\ Tu szuka ciżba, tysiącami mnoga, \\ Zbiegłych Dryjadom i Faunom uśmiechów. \\ Tu wszystko czerstwi, weseli, zachwyca, \\ Czy ciągnę tchnienie, co się zimnem czyści, \\ Czy na niebieskie zmysł podniosę lica, \\ Czyli się śnieżnej przypatruję kiści $[\ldots]^{32}$
}

[Willkommen! Den Stadtvölkern eine glückselige Zeit, / Sowohl den Memel-Leuten als auch den Nachbarlechen / Sucht hier bereits das Gewimmel auf, mit Tausend / Lächeln von Driaden und Frauen. // Alles hier erheitert, erfreut, begeistert, / Entweder ziehe ich

31 MickiewiCz (wie Anm. 25), Bd. 1, 15.

32 Ebd. 
den Hauch, gereinigt durch die Kälte / Oder ich erhebe die Sinne auf die himmlischen Antlitze auf das Blaue/ Oder ich betrachte die Schneetrauben...]

Charakteristisch ist, dass der Winter bzw. die Winteridylle hier zwar lokalisiert, aber noch nicht explizit nationalisiert wird; sie gehört sowohl den Litauern bzw. Litvinen (Niemeńcy), als auch den Polen (Lechi), die - der Ideologie des polnisch-litauischen metonymischen Partialitätsschreibens entsprechend - komplementär und zugleich synonym auftreten. Nicht zu überhören ist auch die leicht ironische Intonation des Gedichts, die im Reim Lechów - uśmiechów entblößt wird. ${ }^{33}$

Eine andere, mit einem heiteren Humor unterstützte Abgrenzung vollzieht sich im weiteren Verlauf des Gedichts; der Monotonie des Winters auf dem Land wird die städtische Winteridylle gegenübergestellt; ${ }^{34}$ dabei werden die Topoi der neoklassizistischen mediterranistischen (Winter-)Pastorale wiedergegeben und zugleich ironisiert. Der Held des Gedichts, der auch für den neuen bzw. noch zu schaffenden Helden der neuen Literatur steht, flieht in die Stadt. Metaliterarisch gelesen, umschreibt diese Flucht des Erzählers Mickiewicz' eine Abkehr von den automatisierten neoklassizistischen Formeln und Diktionen, die in den Strophen zuvor artikuliert wurden (Plutos, Ceres, Chariten). Dieser programmatische Verzicht geschieht zugunsten eines neuen Themas, nämlich des orientalischen. Für die Verschränkung der sich einerseits selbst nordisierenden

33 Man kann nur vermuten bzw. spekulieren, dass (ob) Mickiewicz, der zur Zeit der Niederschrift des Gedichts Deutsch gelernt hat, von der im Deutschen vorhandenen Paronymie Lächeln (uśmiech) zu Lech zu seinem Reim inspiriert wurde.

34 In der in „Zima miejska“ zentralen Gegenüberstellung von Stadt und Land sind im Rahmen des Wintersujets die ersten Ansätze der Spaltung bzw. der Differenzierung unterschiedlicher Winterbilder zu beobachten, die Mickiewicz später bei seiner Dekonstruktion des russischen Winters im „Ustęp“ [Exkurs, Digression] zum III. Teil des Poems „Dziady“ autotextuell zu Hilfe kommen. Vgl. postkolonialistische Interpretationen des Russlandbildes in Mickiewicz' „Ustęp“ bei GALL, Alfred: Konfrontation mit dem Imperium: Die literarische Auseinandersetzung zwischen Adam Mickiewicz („Dziady“) und Aleksandr S. Puškin („Mednyj vsadnik“ [Eherner Reiter]). In: Die Slaven und Europa. Hg. v. Gerhard RESSEL und Henrieke STAHL. Frankfurt am Main u.a. 2008 (Trierer Abhandlungen zur Slavistik 8), 79-103 und KIRSCHBAUM, Heinrich: Die Staatsgrenzen der Romantik: Zur Funktion des ,grenzenlosen Russlands' bei Adam Mickiewicz. In: Grenzen. Konstruktionen und Bedeutungen. Hg. v. Dennis GRÄF und Verena SchmöLler. Passau 2009 (Medien, Texte, Semiotik Passau 2), 211-234. 
Diktion (polnisch-litauische Winteridylle) und den $\mathrm{zu}$ dekonstruierenden neoklassizistisch-mediterranistischen Topoi mit den orientalistischen ist von Relevanz, dass bei der Beschreibung des Tagesablaufes des lyrischen Helden - des jungen Wilnaer Dandys - zunehmend orientalische bzw. asiatische Realia erscheinen. ${ }^{35}$ Chinesische Objekte - nankin (Nanking-Schlafrock), chińskie zioła (chinesische Kräuter) - stehen neben den türkischen - stambulskie gorycze (Istanbuler Bitterkeiten [Tabak]) und dem orientalischen Balsam (wschodni balsam). Hier differenziert Mickiewicz noch nicht zwischen den unterschiedlichen östlichen Realia, wichtig ist nur, dass sie aus dem Osten kommen. Das Besingen des Winters und des Nordens schließt Orientalismen und Selbstorientalisierungen nicht aus, wobei diese Selbstorientalisierungen Teil der eigenen SelbstUrbanisierungs- und somit Selbsteuropäisierungs- und Selbstzivilisierungsstrategien des aus der Provinz stammenden Dichters (und seines autobiographischen Helden) bedeutet. Die Exotismen des orientalisierten Morgenalltags eines neuen Stadtdandys gehen harmonisch, ja idyllisch in die Beschreibungen des Stadtwinters über. ${ }^{36}$ Nach dem Besuch eines Salons bzw. eines Klubs (Mickiewicz konstruiert eine Sammelbeschreibung des städtischen Alltags der Wilnaer Junggesellen) fährt man nach Hause. Die schönen Winterbilder, mit denen das Gedicht begann, schließen es auch $\mathrm{ab}^{37}$. Der Winter dient Mickiewicz nicht nur als (Nord-)Kulisse, sondern auch als Verkörperung der Jugend im Allgemeinen und der poetischen Jugend im Einzelnen. Die Selbstnordisierung bildet in „Zima miejska“ nicht nur die Leitdiktion des Gedichts (vgl. das quantitativ und qualitativ dominierende semantische Feld des Winters: Schnee, Eis, Kälte etc.), sondern auch dessen Rahmenkonstruktion, innerhalb derer eine Wende vom neoklassizistisch-mediterranistischen Schreiben zum orientalistischen symbolisch beschrieben wird.

Zur Intensivierung der Beschäftigung mit dem Orient bei Mickiewicz kommt es noch vor seiner Verbannung nach Russland und noch vor seiner Reise auf die Krim. Im September 1824 schreibt bzw. improvisiert Mickiewicz im Wilnaer Gefängnis die „türkische Ballade“ (ballada turecka) „Renegat“. ${ }^{38}$ Die Auftaktverse der Ballade klingen metapoetisch: Es ist für den Erzähler Zeit, über die Ereignisse im Iran zu berichten und für Mickiewicz ist es Zeit, sich des orientalischen Themas zu bedienen:

35 Mickiewicz (wie Anm. 25), Bd. 1, 16.

36 Ebd.

37 Mickiewicz (wie Anm. 25), Bd. 1, 17.

38 „Renegat“ und „Zima miejska“ weisen direkte autotextuelle Konvergenzen auf: Vgl. das in den beiden Texten vorhandene Motiv des Kaschmirteppichs. 
Co się niedawno stało w Iranie,

Opowiem światu całemu:

Na kaszemirskim usiadł dywanie

Basza pośrodku haremu. ${ }^{39}$

[Was neuerlich im Iran geschah, / Erzähle ich der ganzen Welt: / Auf dem Kaschmirteppich / Saß der Pascha inmitten des Harems.]

Mickiewicz schafft in seinem Gedicht ein orientalistisches Super- und Metasujet. Das Gedicht ist beinahe überfüllt von orientalischen Exotismen, hier in der Reihenfolge ihres Erscheinens (einige erscheinen mehrmals): Iran, kaszemirski $d y$ wan (Kaschmir-Teppich), harem (Harem), padiszach (Padischah), Czerkieski (Tscherkessinnen), Kirgis (Kirgise), Eblis (Iblis bzw. Azazil), basza (Pascha), turban (Turban), cybuch (Tabakpfeife), Kyzlar-Aga, Effendy (Effendi), Stambul (Istanbul), janczary (Janitschar), Nazaretka (Nazaretherin), Hassan, tygrys (Tiger), Dżurdżistan (Dschurdistan [Georgien]), hurysa (Huris), Chan (Khan), Chagan (Kaghan), gazel (Gazelle), bej (Bej), etc.

Für ein solches orientalistisches Sammelbild eignete sich am besten das damals populäre Haremsujet, in dem per definitionem unterschiedliche Personen und Realien aus der ganzen (orientalischen) Welt vertreten sein müssen. Sogar Polen (bzw. Litauen) wird türkisch und somit selbst-orientalisierend als Lehistan bezeichnet. ${ }^{40}$ Die übertriebene Konzentriertheit der orientalischen Realia wirkt ironisch, was der ganzen Ballade einen leicht parodistischen Ton verleiht. Der Ort der Parodie wird allerdings zum Experimentierfeld für spätere Poetisierungen bereits jenseits des Parodistischen (wie in „Sonety Krymskie“ [Krimsonette] von 1826). Zwar wird Polen orientalisiert, zugleich aber in Opposition zum Orient gestellt. Der Mehrdeutigkeit und Multifunktionalität seiner Selbstorientalisierungen bleibt Mickiewicz auch in seinem Spätwerk treu.

Basza nie widzi, basza nie słucha,

Turban zawiesił nad okiem,

Drzemie i dymy ciągnąc z cybucha

Okrył się wonnym obłokiem. ${ }^{41}$

39 Mickiewicz (wie Anm. 25), Bd. 1, 195.

40 Ebd.

41 Ebd. 
[Der Pascha sieht nicht, der Pascha hört nicht, / Der Turban über dem Auge gehängt, / Schlummert er, und den Rauch aus der Pfeife ziehend, / Bedeckte er sich mit einer Wolke.]

Der Pascha, der hier symbolisch, metaliterarisch gelesen, für den Orientalismus steht, ist der alten orientalischen (und metaliterarisch gelesen orientalistischen) Schönheiten überdrüssig geworden. Dem Pascha wird eine neue Gefangene vorgeführt, nämlich eine Polin, die wie der Pascha selbst aus kalten Ländern kommt („Ona jest rodem z zimnej krainy“) und aus denen auch der Pascha stammt. Nicht einmal der Padischah habe in seinem Garten der Pracht in Istanbul (,w sadzie rozkoszy w Stambule") eine solche Schönheit. ${ }^{42}$

Der Pascha selbst ist in Wirklichkeit ein Renegat, der einst die Fronten bzw. die Religion gewechselt hat; die Geschichte des Überlaufens wird allerdings nicht erzählt: Mickiewicz mag sich darunter auch einen Janitscharen im kulturhistorischen Sinne des Wortes vorgestellt haben. ${ }^{43}$ Die nordische, polnische (litauische) Schönheit kommt aus dem Heimatland des Überläufers, aus dem Land, das er einmal verlassen (verraten) hat und an das er oft zärtlich dachte. ${ }^{44}$ Metaliterarisch gelesen stellt eine solche Einführung des Einheimischen und Europäischen in Einem (beim Motiv des Renegatentums und in der Figur der entführten schönen Polin, der Vorläuferin der Maria Potocka aus den „Sonety Krymskie“) eine Europäisierung und zugleich Polonisierung des orientalistischen Sujets dar, die jedoch auch eine Selbstorientalisierung des Europäischen/Nordischen/Polnischen/Litauischen mit sich bringt. Das Europäische, das sich beim ,traditionellen' Orientalismus als maskulin präsentiert, wird nun feminisiert. Die aktuelle Stufe des Orientalismus - diejenige der Faszination - verlangt nach neuen Konstellationen und Verteilungen der (Geschlechter-)Rollen. Somit wird das Haremsujet des europäischen Orientalismus entautomatisiert und erneuert, und zwar durch dessen Polonisierung. In „Renegat“ finden die diskursiven Verquickungen des polnischen und orientalischen Sujets noch in spielerisch-parodistisch anmu-

42 Mickiewicz (wie Anm. 25), Bd. 1, 196.

43 Das Wort Janitschar (janczar) taucht in „Renegat“ auch auf (vgl. die erste Strophe des Teils II), allerdings wohl in der damals geläufigeren Reduzierung der Wortbedeutung auf ,grausamer Angehöriger aus der Garde“.

44 Die Tatsache, dass der Pascha zärtlich melancholisch an seine Heimat dachte, bringt näher, das es sich beim Renegaten wohl kaum um einen kaltblütigen Verräter handelt, sondern um einen, der mehr oder weniger wegen äußeren Umständen (Entführung) seine Heimat verlassen musste, d.h. eher für einen Janitscharen. 
tendem Ton statt. Der Pascha, der Renegat stirbt beim Anblick der neuen Schönheit aus den Mitternachtsländern. ${ }^{45}$

Die Polin als Vertreterin des Nordens bringt sowohl Unruhe in den Harem als auch etwas Neues, einen neuen (thematischen) Zauber (czary), etwas bzw. jemand, den der Pascha, hier stellvertretend für den Orientalismus, bisher nicht gesehen hat. Die alte, ,rein' orientalische Welt des europäischen Orientalismus reicht nicht mehr aus. Der Pascha (lies: der (europäische) Orientalismus) erliegt dieser neuen Strömung. Die Schlange (żmija) des polnischen Sujets schleicht sich darin ein. ${ }^{46}$ Indirekt wird auch das Polnische als Bestandteil des Orientalismus behauptet und als dessen integraler Teil proklamiert. Mehr noch: Durch die Einführung der polnischen Komponente behauptet Mickiewicz sein Recht bzw. das Recht der polnischen Literatur auf poetische, romantische Orientalismen, das Recht, das in deren Sujets selbst angelegt ist. So meistert Mickiewicz das Hauptdilemma der einzelnen, nationalen bzw. sich selbst als national zu behauptenden europäischen Literaturen: Er schreibt sich in das Paradigma der europäischen literarischen Orientalismen ein, behauptet jedoch zugleich seine eigene Originalität. In „Renegat“ geschieht die Selbstbehauptung zum einen durch die Hypertrophie der europäischen romantisch-orientalischen Sujets, zum anderen durch deren ,Polonisierung‘. Es kommt dabei zur Verschränkung von orientalistischer Diktion eines neuen europäischen und zugleich polnischen Romantikers und seiner Selbstorientalisierung, die den ,Preis‘ für die Polonisierung des orientalischen Sujets ausmacht. Zugleich vollzieht sich eine Übertragung des orientalischen Sujets in den Norden, der dadurch zum festen Bestandteil des Orientalismus wird. Die polnische Schönheit flieht in der Begleitung eines bej nach Polen. $^{47}$

Eine solche Entwicklung der Erzählung ermöglicht auch eine weitere Interpretation. Der Renegat erliegt der polnischen, einheimischen Schönheit, die er vielleicht auch früher gekannt hat: Man soll dem zu exotischen Orientalismus den Rücken kehren und eigene, nordische Sujets entwickeln. Im dritten Teil des Gedichts kommt es zu einer weiteren Umschreibung dieser Verschiebung des orientalen Sujets. Die Polin kehrt nach Hause zurück, kann aber ohne ihren Geliebten nicht mehr leben, stirbt und wird begraben. Bei der Trauermesse erscheint allerdings ein Türke und bringt die Botschaft vom verstorbenen Pascha: Sein letzte Wille war, dass er neben seiner Liebe begraben werde und dass die Ringe der beiden ausgetauscht werden. Dass der Pascha ein paar Strophen früher

45 Mickiewicz (wie Anm. 25), Bd. 1, 196.

46 Ebd, 197.

47 Ebd. 
plötzlich verstorben ist, fällt hier nicht mehr ins Gewicht. Es kommt zu einer nicht ohne parodistische Züge - Hypertrophierung und Forcierung unterschiedlicher romantischer Schemata. Es soll nach dem Tode des Paschas zur Vermählung der beiden kommen. Der katholische Priester weigert sich allerdings, den Willen des Paschas zu erfüllen, da dieser ein Renegat ist. Der Türke setzt trotzdem den Ring auf den Finger der Verstorbenen; vergeblich versucht der Priester ihn ihr zu nehmen, die tote Schönheit drückt ihre Hand fest zusammen. Die Rückkehr des Renegaten nach Polen ist als thematische Rückkehr in den heimischen Norden zu interpretieren. Mickiewicz verschiebt die Achse des orientalischen Sujets nach Norden und behauptet im gleichen Schritt die reziproke Abhängigkeit und Verwobenheit der beiden literarischen Diskurse (Orientalismus und Nordismus).

Wenn man die autotextuelle Zukunft dieses Gedichts in Mickiewicz' Werk in Betracht zieht, denkt man neben den „Krimsonetten“, in denen zahlreiche Motive des „Renegat“ weiter verfolgt werden, u.a. an die Entwicklung der Renegatenfigur selbst. In diesem Gedicht wird, obgleich in einer humoresken Form, das Phänomen des Renegatentums angesprochen, das in Mickiewicz' Werk eine der magistralsten Rollen spielen wird und schließlich in die Gestalt des litauischen ,Janitscharen' Konrad Wallenrod münden wird. Dabei sollte nicht vergessen werden, dass „Renegat“ im Gefängnis geschrieben wurde: Es trägt somit zumindest in seiner Entstehungsgeschichte eine bittere Ironie in sich. In „,Konrad Wallenrod“ wird der heitere Galgenhumor des „Renegat“ ins sardonische Lachen des Hauptprotagonisten wechseln.

Das Gedicht „Renegat“, wie auch „Zima miejska“ und andere „unschuldige“, ironische und selbstironische Texte des frühen Mickiewicz, in denen das neoossianistische und orientalistische Schreiben karikierend protokolliert wird, werden zum autotextuellen Laboratorium, aus dem Mickiewicz später seine Bilder und Diktionen, diese umkodierend, neu einsetzen wird. In Folge der zunehmenden Politisierung von Mickiewicz' Diktionen in der zweiten Hälfte der 1820er Jahre werden diese autotextuellen Fertigteile aufgegriffen, zum Teil enthumorisiert und (politisch) ernst eingesetzt.

Die Tatsache, dass mit dem Orientalismus in den beiden besprochenen Texten noch spielerisch umgegangen wird, soll nicht über die zunehmende Intensität der Beschäftigung Mickiewicz' mit dem orientalischen Thema hinwegtäuschen. Bereits während seiner Wilnaer Zeit freundete sich Mickiewicz mit vielen Polen an, die in den folgenden Jahren zu Leitfiguren der russisch-polnischen Orientalistik wurden (Aleksander Chodźko, Józef Szczepan Kowalewski, Józef Sękows- 
ki, Jan Nepomucen Wiernikowski u.a.). ${ }^{48}$ Im November 1824 kommt Mickiewicz in Sankt Petersburg an. Dort begegnet er dem Orientalisten Józef Julian Sękowski (1800-1858). ${ }^{49}$ Sękowski gehörte dabei zu den wenigen Polen, denen der polnische Dichter skeptisch gegenüberstand. ${ }^{50}$ Im Winter 1824/25 besuchte Mickiewicz Sękowskis Haus und schrieb in das Album seiner Frau das Gedicht „Wschód i północ“ [Der Osten und der Norden], in dem er bereits im Titel die hier interessierende geokulturelle Verbindung zwischen dem Orient und dem Norden artikuliert:

Porzuceni na świata lodowatym końcu,

Nie zazdrośćmy krainom sąsiedniejszym słońcu:

Ich ląd kaszemirskiego ma barwę kobierca,

Kwiat z jedwabiu jutrzenki, z płomienia ich serca;

Lecz niech bulbul zabłyśnie - i wnet oko zmruży;

Wnet znikną w ziemi liście, w sercach pamięć róży.

A nasze lądy zimnym dochowują łonem

Pamięć istot straconych przed lat milijonem.

Jeśli ziemia tak długo chowa martwy szczątek,

Jekież są nasze serca - dla żywych pamiątek! $!^{51}$

$48 \mathrm{Zu}$ den Beziehungen Mickiewicz' zu seinen Freunden aus den Wilna-Petersburger orientalistischen Kreisen vgl. ZAJĄCZKOWSKI, Ananiasz: Orient jako źródło inspiracji w literaturze romantycznej doby Mickiewiczowskiej [Der schöpferische Einfluss des Orients auf die romantische Literatur zur Zeit Mickiewicz']. Warszawa 1955.

49 Zum Stellenwert Sękowskis in der russischen (bzw. russisch-polnischen) Osmanistik und Iranistik vgl. Istorija otečestvennogo vostokovedenija do serediny XIX veka [Geschichte der russischen Asienkunde bis zur Mitte des 19. Jahrhunderts]. Hg. v. Georgij KIM. Moskva 1990, 202-206, 209, 212f. Zu den Identitätsstrategien Sękowskis vgl. auch GroB, Thomas: Metafiktionalität, Nullpunkt und Melancholie. Osip Senkovskijs „Phantastische Reisen des Baron Brambeus“ am ,Ende“ der Romantik. In: Romantik, Moderne, Postmoderne: Beiträge zum ersten Kolloquium des Jungen Forums Slavistische Literaturwissenschaft. Hg. v. Christine GöLZ u.a. Frankfurt am Main 1998 (Slavische Literaturen 15), 66-97 und GROB, Thomas: Autormystifikation, kommunikatives Framing und gespaltener Diskurs. Baron Brambeus als ,postromantische" metadiskursive Konstruktion. In: Mystifikation - Autorschaft - Original. Hg. v. Susi FRANK u.a. Tübingen 2001 (Literatur und Anthropologie 9), 107-134.

50 Vgl. Mickiewicz (wie Anm. 25), Bd. 4, 452.

51 MicKiewicz (wie Anm. 25), Bd. 1, 209. 
[Verlassen am Ende der eisigen Welt, / Beneiden wir nicht die Nachbarländer der Sonne: / Ihr Land hat die Farbe des Kaschmirteppichs, / Die Blume aus der Seide der Morgenröte, aus der Flamme seines [des Landes - H.K.] Herzens; / Blitzt jedoch der Bülbül auf - und gleich schließt er das Auge; / Gleich verschwinden in der Erde die Blätter, in den Herzen das Gedächtnis der Rose. / Und unser Eis wahrt mit seinem kalten Schoß / Das Gedächtnis der verlorenen Geschöpfe vor Millionen Jahren. / Wenn die Erde schon so lange tote Trümmer aufbewahrt, / Sind unsere Herzen - für lebendige Erinnerungen].

Die Metonymie Orient - Norden polarisiert sich hier in eine Dichotomie. Über das Bild des Kaschmirteppichs (kaszemirski kobierzec) schlägt Mickiewicz eine explizite autotextuelle Verbindung zu seinem wenige Monate zuvor geschriebenem Gedicht „Renegat“ (kaszemirski dywan). Im Gegensatz zu „Renegat“ unterstreichen die orientalischen Fremdwörter und Exotismen jedoch in „Wschód i północ" die Fremdheit der hinter ihnen stehenden (poetischen) Realien. Das Gedicht erhält eine zusätzliche poetologische, metaliterarische Dimension durch die Einführung des Motivs der Nachtigall - bulbul. Dem ,klassischen“ orientalistischen Motiv (Nachtigall - Rose) wird das Gedächtnis der Erde gegenüber gestellt. In „Wschód i północ“ nimmt Mickiewicz Abstand sowohl zu seinen marginalen, dekorativen Exotismen des europäischen Städteralltags in „Zima miejska“" als auch zu dem hypertrophierten Orientalismus in dem orientalistischnordistischen Programm von „Renegat“. Die Argumentation dieser Abgrenzung, ,biographisch“ motiviert durch die Abneigung gegenüber dem ,Renegaten“, dem Orientalisten Sękowski, der in den Augen Mickiewicz' den Orient gegen den Norden (d.h. Polen bzw. Polentum) getauscht habe, ist folgende: Hinter dem Norden steht im Gegensatz zum fremden exotischen Orient das Historische im Allgemeinen und das kulturelle Gedächtnis des eigenen Volkes im Besonderen. Nur zur Erinnerung: Seinen Eid gegenüber dem kalten winterlichen Norden schwört ein Dichter, der kurz zuvor nicht nur an seinen litauischen Balladen, sondern auch an seinem Poem „Dziady“ gearbeitet hat, in dem bereits im Titel der Ahnenkult und die Vorfahrenfeier zum Programm wird. In der Topik der Erinnerung an die Ahnen verschränken sich bei Mickiewicz das politische Pathos des kulturellen Gedächtnisses der gespaltenen Nation und die post-ossianistischen Sujets vom Getrennt- und Gespalten-sein, ja vom postkolonialistisch $\mathrm{zu}$ verstehenden Dazwischen (inbetweenness) des noch nicht Toten und noch nicht und zugleich nicht mehr Lebendigen in der (Traum-)Wirklichkeit der Erinnerung.

Das Historisch-Patriotische fehlt dem Orientalismus trotz all seiner (literarischen) Attraktivität: Später wird Mickiewicz dieses Dilemma lösen, indem er das Nordisch-Litauische und das Orientalische, mal kontrastiv, mal interferierend in 
Relation zu einander bringen und sich selbst orientalisierend und nordisierend, von der Trennung des polnischen Dichters von seiner litauischen Heimat (Geliebten) berichten wird. Auf der Reise durch den ukrainischen, vormalig polnischen Süden Russlands wird der „Litauer“ Mickiewicz persönlich - ja biographisch bzw. seine eigene romantische Biographie kreierend - dem Orient begegnen und anschließend vor der intertextuellen Folie von Puškins „Bachčisarajskij fontan“ seine „Sonety Krymskie“ schreiben, in denen er eine im Vergleich zu den oben besprochenen Gedichten komplexere antiimperiale und zugleich koloniale Metonymie Litauen (Polen) - Orient konstruieren und somit eine neue orientalistisch-ossianistische und zugleich sich selbst orientalisierende poetischpolitische Identität entwerfen wird. ${ }^{52}$

Orientalismus und Ossianismus, die beiden Diskursdoppelgänger, werden später, Anfang der 1830er Jahre, nach der Enttäuschung über die Folgen des Novemberaufstandes - auf einem neuen Niveau - zu ihrem dritten diskursiven Doppelgänger, ja zum ,Original“ zurückkehren, von dem in „Zima miejska“ Abschied genommen wurde: zur neoklassizistischen mediterranistischen Idylle, und zwar in der mit Humor und bitterer, aber zugleich heiterer Selbstironie entworfenen Halb-Ballade, Halb-Pastorale von „Pan Tadeusz“ (1834). Dort wird Mickiewicz, seine Erfahrungen des nordistisch-orientalistischen Schreibens summierend, dem gespaltenen und voneinander (und, wenn man so sagen darf, miteinander) getrennten polnischen Volk, zugleich jedoch seiner eigenen literarischen Jugend ein poetisches Mahnmal errichten, das die Wege der polnischen (Literatur-)Geschichte für Jahrzehnte maßgeblich mit bestimmen sollte.

52 Vgl. dazu ausführlicher KIRSCHBAUM, Heinrich: Im Harem des Imperiums. „Bachčisarajskij fontan“, „Sonety Krymskie“ und der russisch-polnische Orientalismus. In: Zeitschrift für slavische Philologie 66/2 (2009-2010), 287-316. 
Bereitgestellt von | Universitaetsbibliothek Basel

Angemeldet

Heruntergeladen am | 18.09.18 11:12 